\title{
¿Qué aspectos considerar al iniciar el tratamiento nutricional para Fenilcetonuria?
}

\section{Which aspects should be considered when starting with the Phenylketonuria diet?}

\author{
López-Mejía Lizbeth ${ }^{1 *}$, Vergara-Vázquez Mónica ${ }^{2 *}$, Guillén-López Sara ${ }^{1 * *}$
}

\begin{abstract}
Resumen
La fenilcetonuria clásica (PKU) es una enfermedad genética en la cual el metabolismo del aminoácido esencial fenilalanina (PHE), se ve obstaculizado por la deficiencia de una enzima llamada fenilalanina hidroxilasa, como consecuencia de este bloqueo los niveles de PHE se acumulan en sangre y otros tejidos, causando daños graves e irreversibles a nivel del sistema nervioso central. El objetivo del tratamiento nutricional consiste en proporcionar una cantidad específica de PHE y al mismo tiempo dar la cantidad de proteína que requiere el paciente para crecer y evitar deficiencias asociadas a la eliminación de alimentos altos en proteína, además de mantener un funcionamiento mental adecuado. El tratamiento nutricional inicialmente, requiere del uso de una fórmula sin PHE, que provea al paciente las proteínas que necesita además de vitaminas y nutrimentos inorgánicos. En los pacientes con PKU es necesario ofrecer lactancia materna, sin embargo no puede ser de forma exclusiva ya que debe combinarse con una fórmula libre PHE.

PALABRAS CLAVE: Fenilcetonuria, terapia nutricional, tamiz neonatal, lactancia materna.

\section{Abstract}

Classical phenylketonuria (PKU) is a genetic disease of the phenylalanine (PHE) metabolism, which is hindered by the deficiency of an enzyme called phenylalanine hydroxylase, as a result of this blockage, PHE levels accumulate in the blood and other tissues, causing irreversible damage to the central nervous system.

The goal of nutritional support is to provide a specific amount of PHE and at the same time, meeting protein requirements for patients to grow and avoid nutritional deficiencies associated with the elimination of high protein foods, in addition to maintain a proper mental functioning. Nutritional treatment requires the use of a formula without $\mathrm{PHE}$, to provide proteins, vitamins and minerals. In patients with PKU is necessary to offer breastfeeding, however it should not be an exclusive feeding, it needs to be combined with a free PHE formula.
\end{abstract}

KEYWORDS: Phenylketonuria, nutrition therapy, newborn screening, breastfeeding

\author{
1 Laboratorio de Errores Innatos del \\ Metabolismo y Tamiz. \\ ${ }^{2}$ Dirección de Atención a la Salud Ma- \\ terna y Perinatal. \\ Correspondencia \\ MN Sara Guillén López \\ sara_guillen@hotmail.com \\ Este artículo debe citarse como \\ López MejíaL, Vergara Vázquez M, Gui- \\ llén López $\mathrm{S}$. ¿Qué aspectos considerar \\ al iniciar el tratamiento nutricional \\ para Fenilcetonuria? Acta Pediatr Mex. \\ 2018;SI(39):66S-74S.
}

\section{INTRODUCCIÓN}

La fenilcetonuria Clásica (PKU) es una enfermedad genética en la cual, el metabolismo del aminoácido esencial fenilalanina (PHE), se ve obstaculizado por la deficiencia de una enzima Ilamada fenilalanina hidroxilasa y como conse- cuencia de este bloqueo los niveles de PHE se acumulan en sangre y otros tejidos causando graves daños irreversibles a nivel del sistema nervioso central. ${ }^{1}$

La PHE es un aminoácido aromático esencial necesario para la síntesis de proteínas, tam- 
bién es usado como fuente de energía alterna durante el catabolismo muscular. La tirosina es un aminoácido que en condiciones normales se produce por la hidroxilación de la PHE y es un precursor de la síntesis de melanina y neurotransmisores como dopamina. En la PKU, la tirosina se convierte en un aminoácido condicionalmente esencial ya que no se producen cantidades suficientes de forma endógena y debe ser suplementado cuando los niveles en sangre se encuentren bajos o se sospeche deficiencia por datos clínicos. ${ }^{2}$

Aquellos pacientes que en alguna determinación en sangre de PHE obtengan un resultado mayor a $360 \mathrm{mmol} / \mathrm{L}$ o $6 \mathrm{mg} / \mathrm{dl}$ requerirán un tratamiento dietético, valores más bajos, deberán ser sujetos a un monitoreo continuo. ${ }^{2}$

Uno de los tratamientos más utilizados en esta patología, es el tratamiento nutricional, cuyo objetivo consiste en limitar más no eliminar la PHE proveniente de la dieta, con el objetivo de evitar su acumulación en el cerebro. El manejo dietético es el más frecuentemente utilizado por su costo y accesibilidad. ${ }^{3}$ Existen nuevos tratamientos farmacológicos, como el dihidrocloruro de sapropterina, $\left(\right.$ Kuvan $\left.^{\circledR}\right)$ el cual es cofactor de la enzima fenilalanina hidroxilasa y ha demostrado tener un efecto tanto clínico como bioquímico en pacientes $\mathrm{PKU}$, otro de los tratamientos más recientes, es el uso de la fenilalanina-amonio-liasa pegilada (Peg-PAL, Pegvaliase $\left.{ }^{\circledR}\right) .{ }^{4}$

El objetivo del tratamiento nutricional consiste en proporcionar una cantidad específica de PHE y al mismo tiempo dar la proteína que el paciente requiere, para crecer y evitar deficiencias asociadas a la eliminación de alimentos altos en proteína, además de mantener un funcionamiento mental adecuado. ${ }^{1,5}$ Es posible tener un crecimiento adecuado en peso y talla a lo largo de la vida con el tratamiento dietético restringido en PHE. ${ }^{6}$
La restricción de PHE en la dieta debe ser tal, que el paciente mantenga niveles sanguíneos de este aminoácido en el intervalo de 120 a 360 mmol/L o 2 a $6 \mathrm{mg} / \mathrm{dl}$, además de mantener niveles de tirosina en valores normales durante toda la vida, lo anterior con la finalidad de evitar disminución del coeficiente intelectual, daño neurológico irreversible, convulsiones, problemas del comportamiento, entre otros. ${ }^{2,7}$ Los primeros años de vida para un paciente con PKU son una etapa crucial ya que es un periodo de crecimiento y desarrollo cognitivo muy importante. Se ha visto que durante esta etapa, los niveles sanguíneos de PHE arriba de 6 mg/dl ó $360 \mu \mathrm{mol} / \mathrm{L}$ se relacionan con un menor desarrollo cognitivo, un meta-análisis realizado por Waisbren y cols mostraron que cada aumento de $100 \mu \mathrm{mol} / \mathrm{L}$ de PHE ocasiona una reducción de entre 1.3 a 3.1 puntos en el coeficiente litelectual. ${ }^{8}$ En otro estudio realizado por de la Parra y cols. observaron que mantener un adecuado control metabólico durante el primer año de vida tiene un impacto positivo en el funcionamiento cognitivo durante la infancia, así como en el control metabólico en años posteriores. ${ }^{9}$

\section{Tratamiento de la Fenilcetonuria al diagnóstico}

Cuando el tamiz neonatal es positivo para PKU y se han realizado los estudios confirmatorios, es importante iniciar el tratamiento nutricional de forma inmediata para disminuir los niveles de PHE en sangre al nivel objetivo (menos de $360 \mu \mathrm{mol} / \mathrm{L}$ ) y evitar daño neurológico; las guías europeas recomiendan que el tratamiento comience a más tardar a los 10 días de vida. ${ }^{10}$

Durante las primeras horas después del diagnóstico, dependiendo los niveles iniciales de PHE en sangre, puede ser necesario eliminar toda fuente de PHE de la dieta, ofreciendo únicamente alimento o fórmula médica libre de PHE por un periodo de 24 a 96 horas como máximo. ${ }^{11}$ Con el objetivo de identificar el momento oportuno 
para la reintroducción de $\mathrm{PHE}$, se recomienda tomar muestras en sangre de PHE cada 24 horas, cuando los niveles se acerquen a $360 \mu \mathrm{mol} / \mathrm{L}$, la ingestión de PHE en lactantes se realizará a través de la leche materna o fórmula infantil. En caso de no contar con los resultados cada 24 horas se debe estimar el tiempo que tardará la PHE en disminuir a los rangos esperados para reintroducirla en la dieta, una restricción prolongada tiene repercusiones en el crecimiento y desarroIlo de los niños. ${ }^{11}$ La cantidad de PHE que debe ingerir cada paciente dependerá de la tolerancia individual la cual varía según la edad, estado nutricional, tipo de variante patogénica, actividad enzimática residual, etc. En los primeros meses de vida, la alimentación del paciente se realizará con alimento o fórmula médica sin PHE más una fuente de PHE, la cual será leche materna como primera opción y si esto no fuera posible, se optará por una fórmula infantil de inicio. ${ }^{12}$

\section{El alimento médico sin PHE para PKU}

El tratamiento nutricional requiere del uso de una fórmula sin PHE o también Ilamada alimento médico, que proporcione al paciente las proteínas que necesita además de otros macro y micro nutrimentos. ${ }^{5}$ Existen nuevas opciones de aporte proteico sin PHE o con pocas cantidades de éste, como es el glicomacropéptido y los aminoácidos largos neutros; los cuales son terapias actualmente utilizadas en otros países con buenos resultados y que generalmente se usan en niños y adolescentes. ${ }^{13,}{ }^{14}$ Cuando se realiza el cálculo dietético de las cantidades de fórmula y alimentos que requiere el paciente, ésta se hace en función de las necesidades de proteína que se requiere por edad, tomando como base las recomendaciones específicas de proteína para esta enfermedad. La tabla de recomendación de PHE, TYR, proteína y energía al día se anexa en el Cuadro 1 y sirve como referencia inicial para determinar la cantidad de fórmula médica y leche materna que necesitará
Cuadro 1. Recomendación diaria de fenilalanina, tirosina, proteína y energía para pacientes con Fenilcetonuria clásica. $15,16,17$

\begin{tabular}{l|c|c|c|c|}
\multicolumn{5}{|c}{ Recomendación diaria de PHE, TYR energía para } \\
pacientes con PKU
\end{tabular}

el paciente para mantener un adecuado estado de nutrición ${ }^{15,16,17}$, sin embargo posteriormente es necesario ir modificando la cantidad de PHE según la tolerancia del paciente. ${ }^{18}$

A nivel mundial existen diversas fórmulas metabólicas libres de PHE, cuya composición varía en la cantidad de macro y micronutrimentos; algunas no contienen lípidos (PhenylAde aminoacid blend ${ }^{\circledR}$, PKU Drink ${ }^{\circledR}$, Lophlex Powder $^{\circledR}$, PKU cooler $^{\circledR}$, PKU express ${ }^{\circledR}$ ) otras no contienen ácidos grasos esenciales como el Ácido Docosahexaenoico (DHA) (Phenex 1 y $\left.2^{\circledR}\right)$, y otras sí lo contienen. (Periflex Junior Plus ${ }^{\circledR}$, PKU Periflex Early years $\left.{ }^{\circledR}\right) .{ }^{15}$

Las fórmulas médicas sin PHE se encuentran incluidas en el cuadro básico del Catálogo de Medicamentos del Consejo de Salubridad Ge- 
neral, la fórmula de inicio libre de PHE tiene la clave 010.000 .5400 .00 y la fórmula de seguimiento libre de PHE la 010.000.5401.00. En México solamente existen tres compañías que ofertan fórmulas libres de PHE: Phenex- $1^{\circledR}$ para recién nacidos y niños, Phenex-2 para niños y adultos, ambas de la empresa Abbott ${ }^{\circledR}$, "Alfamir libre de fenilalanina" ${ }^{\circledR}$ para niños mayores de 8 años y adultos de Nucitec $^{\circledR}$, "Beu 2 Intoleranz Fórmula de seguimiento Libre de Fenilalanina ${ }^{\circledR \prime}$ para niños de 8 años a adultos de laboratorios Fracaâ. Actualmente solo existe una presentación de fórmula metabólica sin PHE para lactantes o para el primer año de vida, en el Cuadro 2 se proporcionan recomendaciones prácticas para el pediatra y el personal de salud con respecto a las diferentes fórmulas infantiles que existen en el mercado, así como sus indicaciones o contraindicaciones en esta enfermedad. ${ }^{3,11,19}$

\section{La mejor fuente de PHE en los primeros meses: Lactancia materna}

Son ampliamente conocidos los múltiples beneficios de la lactancia materna sobre los lactantes y las madres; en niños sanos se recomienda ofrecerla de forma exclusiva los primeros 6 meses de edad y complementaria hasta los 2 años. En los pacientes con PKU no es posible ofrecer lactancia materna exclusiva, sin embargo, no debe suspenderse sino complementarse con una fórmula libre PHE. Existe evidencia de que los pacientes con PKU alimentados con seno materno mantuvieron un adecuado incremento

Cuadro 2. Recomendaciones prácticas para el uso de diferentes fórmulas en la PKU.,11,19

\section{Recomendaciones prácticas para el uso de diferentes fórmulas en la PKU}

No se recomienda suspender el aporte de PHE por más de 96 horas o 4 días ya que el paciente puede empezar a presentar diversos problemas de salud relacionados con la deficiencia de este aminoácido esencial.

La leche materna es la primera elección como fuente de PHE, ya que a diferencia de fórmulas de infantiles contiene menores cantidades de PHE.

Si por algún motivo no es posible dar seno materno se debe optar por la fórmula de inicio independientemente del peso y edad del paciente ya que en comparación con la fórmula de seguimiento ésta contiene menor cantidad de proteína y por lo tanto de PHE.

En caso de no contar con fórmula metabólica sin PHE de inicio se puede utilizar la versión 2, solamente es necesario considerar que por lo general éstas contienen el doble de proteína y menor cantidad de energía que la versión 1 y otra osmolaridad por gramo, por lo que es necesario hacer un cálculo específico para poder proporcionar todo el aporte calórico.

El uso de fórmula de soya en PKU no es recomendado debido a que, si bien su origen es vegetal, está adicionada con todos los aminoácidos esenciales incluida la PHE y en ocasiones esta cantidad puede llegar a ser mayor que en una fórmula de inicio y claramente más elevada que en el seno materno.

A menos que el paciente con PKU tenga una clara indicación médico-nutricional de una fórmula semielemental o elemental como fuente de PHE, éstas están contraindicadas por contener cantidades elevadas de PHE en comparación con el seno materno o la fórmula de inicio. No ofrecen ninguna ventaja a un paciente con PKU, por el contrario pueden promover una elevación en sangre de PHE completamente indeseable y difícil de controlar.

La alimentación de una madre lactante no deberá de cambiar por tener a un hijo con PKU.

La fórmula para prematuros puede ser usada siempre tomando en cuenta el perfil de aminoácidos que contiene para poder proporcionar al paciente la cantidad que requiere, por lo general estas fórmulas contienen mayores cantidades de PHE y proteína que el seno materno o fórmula de inicio.

La leche entera, de crecimiento, para niños de 1-3 años está contraindicada en menores de 1 año para pacientes con PKU por no contener los nutrimentos que requieren, además de contener una carga de proteína muy importante.

Tomar siempre en cuenta al usar la fórmula metabólica sin PHE, que debido a que contiene aminoácidos libres tiene una mayor carga osmótica, por lo que el cálculo de la osmolaridad debe ser contemplado siempre, en un inicio se puede considerar dar 20 kcal/onza. 
ponderal, así como niveles de PHE más favorables en comparación con aquellos que no recibieron lactancia materna. ${ }^{19}$

Desde hace algunos años han surgido publicaciones referentes al sobrepeso y obesidad en pacientes con $\mathrm{PKU},{ }^{20}$ una de las herramientas de prevención es a través de la lactancia materna, la cual se ha asociado a la disminución en el riesgo de padecer sobrepeso y obesidad y a un coeficiente intelectual más elevado. ${ }^{21,22}$ La leche materna contiene menor cantidad de proteína y por lo tanto de PHE en comparación con fórmulas infantiles, una alta ingestión proteica en la infancia se ha relacionado con un riesgo más alto de obesidad. ${ }^{23}$

Existen diferentes métodos para administrar la leche materna en los pacientes con PKU: 1) proporcionar una cantidad calculada de fórmula médica sin PHE seguida de lactancia materna a libre demanda, 2) extraer leche materna y proporcionar una cantidad calculada seguida de fórmula médica libre de PHE a libre demanda y 3) alternar las tomas al pecho o leche materna extraída y alimentación con fórmula médica libre de PHE. ${ }^{24}$ Es necesario valorar con los padres del paciente la técnica más adecuada para lograr el óptimo control de la PHE en base a las costumbres, hábitos del paciente y su familia. En el caso de un lactante a quien no sea posible ofrecerle lactancia materna, se recomienda como fuente de proteína intacta fórmula infantil de inicio, la cual puede administrarse antes o después de la fórmula médica sin PHE o bien pueden mezclarse en un una misma toma. ${ }^{22}$ Existen pequeñas diferencias en las cantidades de PHE, que pueden resultar significativas entre las fórmulas infantiles de inicio, por lo que contar con el aminograma de cada una y la cantidad exacta de proteína es esencial. ${ }^{15}$

\section{Alimentación complementaria en Fenilcetonuria}

La alimentación complementaria es un proceso crucial para el desarrollo de los lactantes; la Organización Mundial de la Salud (OMS) recomienda que la introducción de alimentos se inicie a partir de los 6 meses de edad, esta recomendación aplica también para los pacientes con PKU, sin embargo Mac Donald A, et al. consideran que introducir sólidos un poco antes de los 6 meses de vida, a los 5 meses, puede ser benéfico, ya que existe menor respuesta neofóbica a los alimentos, y posiblemente lleve a una mejor aceptación de la comida. ${ }^{25}$ La decisión del momento para iniciar la alimentación complementaria debe ser individualizado ya que se deben tomar en cuenta los hitos del desarrollo como: adecuado sostén cefálico, disminución de la protrusión de la lengua, desarrollo de movimientos de mano a boca, función neurológica, entre otros, sin embargo se recomienda que no sea antes de las 17 semana ni después de las 26 semanas de vida. ${ }^{15,26}$ La alimentación complementaria en PKU se inicia con verduras, frutas y cereales en cantidades específicas previamente calculadas para ofrecer una cantidad determinada de PHE que tolere el paciente $y$ mantenga sus niveles de PHE por debajo de 360 $\mu \mathrm{mol} / \mathrm{L}$. En un principio la cantidad de alimento que se introduce es pequeña por lo que la leche materna o fórmula infantil siguen siendo la principal fuente de PHE de la dieta, conforme se introduce una mayor cantidad de alimento a la dieta del lactante, la PHE de la leche materna o fórmula infantil se reemplaza por la PHE de estos alimentos. ${ }^{25}$ La fórmula libre de PHE se mantiene y ajusta en esta etapa, nunca debe suspenderse, pues durante toda la vida será la principal fuente de proteína y energía de los pacientes. ${ }^{15}$

En PKU la introducción de alimentos requiere de especial atención ya que los padres además de aprender el proceso de introducción de alimentos habitual, requieren de un entrenamiento especial para aprender a cuantificar la PHE o cantidad de proteína que ingieren sus hijos, por lo que la intervención de un nutriólogo especialista en PKU es fundamental. ${ }^{25}$ Para cuantificar 
la cantidad de PHE y proteína existen tablas de alimentos agrupados en sistema de equivalentes para facilitar la elección, conteo y variedad en la dieta de éstos pacientes.

\section{Proceso de cuidado nutricio en PKU}

Para un manejo adecuado de estos pacientes, es necesario que desde el momento del diagnóstico y durante toda la vida, se realicen evaluaciones periódicas que incluyan parámetros antropométricos, bioquímicos, clínicos y dietéticos, así como también la interacción fármaco-nutrimento que pudiera existir. ${ }^{15} \mathrm{En}$ el Cuadro 3 se describen los puntos a considerar

Cuadro 3. Evaluación del estado de nutrición en $\mathrm{PKU}^{27}$

\begin{tabular}{|c|c|}
\hline Parámetros & Puntos básicos a considerar \\
\hline Antropométricos & $\begin{array}{l}\text { Evaluar perímetro cefálico, peso, talla } \\
\text { o longitud, talla para la edad, peso } \\
\text { para la talla o IMC, perímetro de } \\
\text { brazo, pliegue cutáneo tricipital. }\end{array}$ \\
\hline Bioquímicos & $\begin{array}{l}\text { PHE, tirosina, cociente PHE/tirosina, } \\
\text { albúmina, prealbúmina, transferrina, } \\
\text { vitamina D, folatos, ácidos grasos } \\
\text { esenciales. }\end{array}$ \\
\hline Clínicos & $\begin{array}{l}\text { Evidencias de deficiencias de tirosina } \\
\text { como color de piel, cabello, presen- } \\
\text { cia de datos como diarrea, estreñi- } \\
\text { miento, vómito. }\end{array}$ \\
\hline Dietéticos & $\begin{array}{l}\text { Historia de ingestión dietética: si } \\
\text { recibió seno materno, fórmula, edad } \\
\text { a la alimentación complementaria, } \\
\text { preferencia, rechazo, alergias alimen- } \\
\text { tarias, dificultades en la alimentación } \\
\text { (mecánicas o de comportamiento), } \\
\text { adherencia al tratamiento, factores } \\
\text { socio-económicos que afecten la } \\
\text { adherencia, contabilización de feni- } \\
\text { lalanina, tirosina, proteína, energía, } \\
\text { micronutrimentos, macronutrimentos } \\
\text { ingestión del alimento médico (can- } \\
\text { tidad, horario, preparación cantidad } \\
\text { de líquido) }\end{array}$ \\
\hline $\begin{array}{l}\text { Interacción } \\
\text { Fármaco } \\
\text { Nutrimento }\end{array}$ & $\begin{array}{l}\text { Tipo de medicamento, dosis, horarios } \\
\text { en los que los ingiere, interacción } \\
\text { de fármacos con alguna vitamina o } \\
\text { micronutrimento en específico. }\end{array}$ \\
\hline
\end{tabular}

en cada rubro. ${ }^{27}$ Cada uno de estos puntos debe ser tomado en cuenta para prescribir un adecuado tratamiento nutricional. Para documentar e identificar objetivamente problemas específicos relacionados con la nutrición y que requieren una intervención inmediata se incluye el Cuadro 4, el cual describe los problemas nutricios más comunes y su causa, así como su relación con la nutrición. ${ }^{28}$ El cuadro se encuentra disponible en formato PES por sus siglas en inglés; el cual fue desarrollado por la Academia de Nutrición y Dietética de Estado Unidos como parte del proceso de cuidado nutricio y que utiliza la terminología de diagnóstico nutricio (Problema), relacionado con (Etiología), evidenciado por (Signos y síntomas), ${ }^{29}$ en este caso se ha adaptado para la evaluación del paciente con PKU. ${ }^{28}$

\section{CONCLUSIÓN}

La PKU es una enfermedad que requiere de un tratamiento nutricional de por vida, el cual restringe la cantidad de PHE que el paciente ingiere, además debe proveer un adecuado aporte de proteína y energía para mantener a los pacientes con cifras de PHE por debajo de 360 $\mu \mathrm{mol} / \mathrm{L}$ y con un adecuado estado nutricional desde el momento del diagnóstico. Como fuente de PHE de primera instancia, se considera la alimentación al seno materno, la cual es la mejor opción, seguida de la fórmula de inicio. Las fórmulas de seguimiento, para prematuro, semielementales y elementales deben usarse con precaución y tomando en cuenta la cantidad de PHE. Para proporcionar la cantidad de proteína que los pacientes necesitan se requiere el uso de fórmulas o alimentos médicos libres de PHE; el proporcionar las cantidades adecuadas de este aminoácido, así como de proteína y energía será de suma importancia para mantener al paciente con un adecuado crecimiento y desarrollo. La alimentación complementaria debe iniciarse entre los 5 y 6 meses de edad, con verdura, fruta y cereales, para ello, es necesario proporcionar 


\begin{tabular}{|c|c|c|}
\hline & Definición & Ejemplos \\
\hline $\begin{array}{l}\text { Problema } \\
\text { (Diagnóstico } \\
\text { nutricional) }\end{array}$ & $\begin{array}{l}\text { Especificar los problemas actuales } \\
\text { relacionados con la nutrición que } \\
\text { se encontraron en la valoración y } \\
\text { que se abordarán con el trata- } \\
\text { miento nutricional. }\end{array}$ & $\begin{array}{l}\text { Ingestión: Ingestión excesiva de proteínas, ingestión de proteínas } \\
\text { o aminoácidos incompatibles con las necesidades (especificar), } \\
\text { consumo excesivo de energía, consumo insuficiente de energía, } \\
\text { consumo excesivo de grasa, consumo insuficiente de grasas, } \\
\text { infusión excesiva de nutrición enteral, infusión insuficiente de } \\
\text { nutrición enteral, prescripción de nutrición enteral inadecuada de } \\
\text { acuerdo a necesidades. } \\
\text { Clínico: Alteración en metabolismo de nutrimentos, alteración de } \\
\text { valores bioquímicos relacionados con la nutrición, interacción } \\
\text { de alimento-medicamento(especificar), tasa de crecimiento por } \\
\text { debajo de lo esperado, bajo peso, sobrepeso u obesidad. } \\
\text { Conductual-ambiental: Falta de conocimiento relacionado con } \\
\text { la alimentación y nutrición, mal apego a las recomendaciones } \\
\text { nutricionales, acceso limitado a alimentos. }\end{array}$ \\
\hline $\begin{array}{l}\text { Etiología } \\
\text { (Relacionado a) }\end{array}$ & $\begin{array}{l}\text { Identificar las principales causas } \\
\text { o factores de riesgo para el } \\
\text { problema específico. La etiología } \\
\text { es el objetivo de la intervención } \\
\text { nutricional. }\end{array}$ & $\begin{array}{l}\text { Factores de consumo: Falta de consumo de fórmula metabólica, } \\
\text { consumo insuficiente de fórmula metabólica, consumo excesivo } \\
\text { de (especificar) } \\
\text { Factores de prescripción: La prescripción nutricional ya no satis- } \\
\text { face las necesidades proteicas, la prescripción nutricional ya no } \\
\text { satisface las necesidades energéticas, prescripción de tratamiento } \\
\text { complementario. } \\
\text { Factores subyacentes a la enfermedad: Nuevo diagnóstico de } \\
\text { PKU, restricción de proteínas / PHE necesaria para el tratamiento, } \\
\text { enfermedad o infección aguda, falta de apetito relacionado a } \\
\text { (especificar) } \\
\text { Factores de conocimiento y comportamiento del paciente/cui- } \\
\text { dador: Elección inadecuada de alimentos, falta de conocimiento, } \\
\text { adherencia inadecuada a las recomendaciones nutricionales, } \\
\text { asistencia a la clínica para iniciar educación nutricional, sin } \\
\text { tratamiento nutricional. } \\
\text { Factores de acceso: Falta de recursos financieros para fórmula } \\
\text { metabólica, falta de seguro para la cobertura de fórmulas metabó- } \\
\text { licas especiales, falta de acceso a los recursos o atención. }\end{array}$ \\
\hline $\begin{array}{l}\text { Signos y síntomas } \\
\text { (Evidenciado por) }\end{array}$ & $\begin{array}{l}\text { Enlistar los datos subjetivos y } \\
\text { objetivos que caracterizan el } \\
\text { problema específico y que se } \\
\text { utilizan usan para monitorizar } \\
\text { la intervención y los resultados } \\
\text { nutricionales. }\end{array}$ & $\begin{array}{l}\text { Valoración bioquímica: Valor de laboratorio comparado con el } \\
\text { valor objetivo (especificar) (por ejemplo, PHE en sangre de } 700 \\
\text { mol /l), aminoácidos elevados (especificar) } \\
\text { Valoración antropométrica: Patrón de crecimiento, peso, peso } \\
\text { para la talla o IMC comparados con los estándares (especificar), } \\
\text { aumento o pérdida ponderal (especificar el cambio de peso) } \\
\text { (especificar el marco de tiempo) } \\
\text { Valoración clínica: Nuevo diagnóstico de PKU, ajustes o nueva } \\
\text { prescripción del tratamiento complementario, deficiencia de } \\
\text { nutrimentos (signo físico o valor de laboratorio) } \\
\text { Valoración dietética: Ingestión estimada a partir del recordatorio } \\
\text { de alimentos comparada con la prescripción dietética (especifi- } \\
\text { que). } \\
\text { Referido por el paciente: Falta de habilidad o comprensión para } \\
\text { implementar la prescripción nutricional, falta de cobertura por la } \\
\text { compañía de seguros para adquirir fórmula metabólica, falta de } \\
\text { apoyo social o familiar. }\end{array}$ \\
\hline
\end{tabular}


educación a la familia con la finalidad de instruirla en el proceso de cuantificación de PHE y proteína en la dieta. Por último en el seguimiento nutricional de estos pacientes, se deben considerar todos los componentes del proceso de cuidado nutricio.

\section{REFERENCIAS}

1. Vockley J, Anderson HC, Antshel KM, Braverman NE, Burton $B K$, Frazier D, et al. Phenylalanine hydroxylase deficiency: diagnosis and management guideline. Genet Med. 2014; 16 (2): 188-200.

2. Sing R, Rohr F, Frazier D, Cunningham A, Mofidi S, Ogata B, et al. Recommendations for the nutrition management of phenylalanine hydroxylase deficiency. Genet Med. 2014; 16(2): 121-131.

3. Giovannini M, Verduci E, Salvatici E, Paci S, Riva E. Phenylketonuria: nutritional advances and challenges. Nutr Metab. 2012; 9(1):7.

4. Sumaily KM, Mujamammi AH. Phenylketonuria: a new look an old topic, advances in laboratory diagnosis, and therapeutic strategies. Int J Health Sci (Qassim). 2017; 11(5):63-70.

5. McLeod E, Ney D. Nutritional management of phenylketonuria. Ann Nestlé Eng. 2010; 68(2): 58-69.

6. Couce ML, Guler I, Anca-Couce A, Lojo M, Mirás A, Leis R, et al. New insights in growth of phenylketonuric patients. Eur J Pediatr. 2015; 174 (5): 651-9.

7. Blau N, van Spronsen FJ, Levy HL. Phenylketonuria. Lancet. 2010; 376 (9750): 1417-27.

8. Waisbren SE, Noel K, Fahrbach K, Cella C, Frame D, Dorenbaum A, et al. Phenylalanine blood levels and clinical outcomes in phenylketonuria: a systematic literature review and meta-analysis. Mol Genet Metab. 2007; 92 (1-2): 63-70.

9. de la Parra A, García MI, Hamilton V, Arias C, Cabello $\mathrm{JF}$, Cornejo V. First year metabolic control guidelines and their impact on future metabolic control and neurocognitive functioning in children with PKU. Mol Genet Metab Rep. 2017; 13: 90-4.

10. Van Wegberg A.M.J., MacDonald A., Ahring K., Belanger-Quintana A., Blau N., Bosch A.M., et al. The complete European guidelines on phenylketonuria: diagnosis and treatment. Orphanet J. Rare Dis. 2017; 12 (1):162

11. Van Calcar S. Phenylketonuria: The diet basis. En: Bernstein L, Rohr F, Helm J, editors. Nutrition management of inherited metabolic diseases. Denver: Springer International publishing. pp 101-10.

12. Banta-Wright SA, Kodadek SM, Steiner RD, Houck GM. Challenges to breastfeeding infants with phenylketonuria. J Pediatr Nurs. 2015; 30(1): 219-26
13. Rocha JC, MacDonald A. Dietary intervention in the management of phenylketonuria: current perspectives. Pediatric Health Med Ther. 2016; 7:155-163.

14. Ney DM, Stroup BM, Clayton MK, Murali SG, Rice GM, Rohr $\mathrm{F}$, et al. Glycomacropeptide for nutritional management of phenylketonuria: a randomized, controlled, crossover trial. Am J Clin Nutr. 2016; 104 (2): 334-45.

15. Acosta P, Matalon KM. Nutrition Management of patients with inherited disorders of aromatic amino acid metabolism. En: Acosta PB, editor. Nutrition Management of patients with inherited metabolic disorders. Sudbury, Massachusetts: Jones and Bartlett publishers; 2010. pp 119-53.

16. Recommended intakes of PHE, TYR and protein for PKU. Southeast Regional Genetics Network SERN. USA 2016. [Citado el 7 de septiembre del 2018]. Disponible en: https://southeastgenetics.org/ngp/guidelines.php/90/ tbls/0/0/PKU\%20Nutrition\%20Guidelines/Version\%20 1.12/List\%20of\%20Tables.

17. Bourges $\mathrm{H}$, Torres $\mathrm{N}$, Tovar A. Proteínas y aminoácidos en: Bourges H, Casanueva E, Rosado JL. Recomendaciones de Ingestión de Nutrimentos para la población Mexicana. Bases Fisiológicas tomo 2. Editorial Médica Panamericana, México, 2008. p. 124.

18. Guillén-López S, Vela-Amieva M. Actualidades en el tratamiento nutricional de la fenilcetonuria. Acta Pediatr Mex. 2011; 32(2):107-114.

19. Kose E, Aksoy B, Kuyum P, Tuncer N, Arslan N, Ozturk Y. The effects of breastfeeding in infants with phenylketonuria. J Pediatr Nurs. 2018; 38: 27-32.

20. Rocha JC, MacDonald A, Trefz F. Is overweight an issue in phenylketonuria? Mol Genet Metab. 2013; 110 Suppl: S18-24.

21. Horta BL, Loret de Mola C, Victoria CG. Breastfeeding and intelligence: a systematic review and meta-analysis. Acta Pediatr. 2015; 104(467): 14-9.

22. Spatz DL. Preventing obesity starts with breastfeeding. J Perinat Neonatal Nurs. 2014; 28(1): 41-50.

23. Tang M. Protein intake during the first two years of life and its association with growth and risk of overweight. Int J Environ Res Public Health. 2018; 15(8). pii: E1742.

24. Pinto A, Adams S, Ahring K, Allen H, Almeida MF, Garcia-Arenas $D$, et al. Early feeding practices in infants with phenylketonuria across Europe. Mol Genet Metab Rep. 2018; 16: 82-89.

25. MacDonald A, Evans S, Cochrane B, Wildgoose J. Weaning infants with phenylketonuria: a review. J Hum Nutr Diet. 2012; 25(2):103-10.

26. Fewtrell M, Bronsky J, Campoy C, Domellöf M, Embleton N, Fidler Mis N, et al. Complementary feeding: a position paper by the European Society for Paediatric Gastroenterology, Hepatology, and Nutrition (ESPGHAN) Committee on Nutrition. J Pediatr Gastroenterol Nutr. 2017: 64(1): 119-132.

27. Nutrition Assesment. Southeast Regional Genetics Network SERN. USA 2016. [Citado el 7 de septiembre del 2018]. 
Disponible en: https://southeastgenetics.org/ngp/guidelines.php/90/npi/0/0/PKU\%20Nutrition\%20Guidelines/ Version\%201.12/Nutrition\%20Problem\%20ldentification.

28. Nutrition problem identification for PKU. Southeast Regional Genetics Network SERN. USA 2016. [Citado el 7 de septiembre del 2018]. Disponible en: https://southeastgenetics.org/ngp/guidelines.php/90/npi/0/0/PKU\%20
Nutrition\%20Guidelines/Version\%201.12/Nutrition\%20 Problem\%20ldentification.

29. Hakel-Smith N, Lewis NM. A standarized nutrition care process and language are essential components of a conceptual model to guide and document nutrition care and patient outcomes. J Am Diet Assoc. 2004; 104 (12): 1878-84. 